\title{
Changing trends in the Antibiotic susceptibility pattern of Acinetobacter species isolated from clinical specimens over a period of five years from a tertiary care hospital
}

\author{
Authors \\ Joana Mary Magdaline ${ }^{1}$, Neethu John ${ }^{2}$, Akhila Aravind ${ }^{3}$ \\ ${ }^{1}$ Associate Professor, Microbiology Dept, Govt. Medical College, Ernakulam, Kerala, India \\ ${ }^{2}$ Assistant Professor, Microbiology Dept, Govt. Medical College Ernakulam, Kerala, India \\ ${ }^{3}$ Junior Resident, Microbiology Dept, Govt. Medical College, Ernakulam, Kerala, India
}

\begin{abstract}
Introduction: Acinetobacter spp has emerged as an important nosocomial pathogen worldwide. Antimicrobial resistance pattern of Acinetobacter vary according to species, country of isolation, region \& period of time. But the overall trend is one of increasing resistance. Hence effective therapy must be individualised to reflect the periodic differences in regional, local \& specific hospital resistance patterns, which makes constant monitoring of antibiogram of Acinetobacter spp essential.

Objectives: 1)To study the prevalence of Acinetobacter spp among various clinical samples over a period of 5 years 2)To assess the prevalence of multidrug resistance among the Acinetobacter isolates 3)To study the changing trends in the susceptibility pattern of Acinetobacter isolates over a period of 5 years

Methodology: Retrospective analysis of the antibiogram of the Acinetobacter spp was done, which were isolated from various clinical specimens received in the Department of Microbiology in a tertiary care centre during a 5 year period from 2012 to 2016

Results: During the study period, a total of 46005 samples were received for culture, from which 583 (1.3\%) Acinetobacter spp were isolated. The prevalence of MDR strains among Acinetobacter increased from $59 \%$ in 2012 to 76\% in 2016. Analysis of the antibiogram of the Acinetobacter isolates showed a progressive decrease in the susceptibility to third generation Cephalosporins \& Piperacillin. The most susceptible agents were Meropenem, Tobramycin \& Cefoperazone-sulbactam.

Conclusion: MDR Acinetobacter infections in our institution have increased considerably over the past five years. The trends in the antibiotic susceptibility patterns needs to be constantly monitored as it will help to determine the emergence of drug resistance, to formulate antibiotic policies for empirical therapy and to curb the irrational usage of antimicrobials.
\end{abstract}

Keywords-Acinetobacter species, Prevalence, Multidrug Resistance, Antibiogram.

\section{Introduction}

Acinetobacter spphave emerged as an important nosocomial pathogen worldwide ${ }^{1}$. They can cause life-threatening infections including pneumonia, bacteraemia, and meningitis and are established as a significant health care problem worldwide ${ }^{1}$.
Antimicrobial resistance pattern of Acinetobacter vary according to species, country of isolation, region and period of time. But the overall trend is one of increasing resistance ${ }^{1}$. Carbapenem resistance is also alarmingly increasing among the Acinetobacter isolates. Hence effective therapy 
must be individualised to reflect the differences in regional, local and specific hospital resistance patterns at regular time intervals. This makes constant monitoring of antibiogram of Acinetobacter spp very essential ${ }^{2}$.

\section{Aims \& Objectives}

1. To study the prevalence of Acinetobacter spp among various clinical samples over a period of 5 years

2. To assess the prevalence of multidrug resistance among the Acinetobacter isolates

3. To study the changing trends in the susceptibility pattern of Acinetobacter isolates over a period of 5 years

\section{Methodology}

The study was conducted in the Department of Microbiology, in a tertiary care centre in Central Kerala. The study included Acinetobacter spp isolated from various clinical specimens in the Department of Microbiology over a period of 5 years, from the year 2012-2016. The antibiogram of the reported Acinetobacter spp in the above mentioned study period were recorded, numerically coded \& entered into Excel spread sheet. An isolate was considered as MDR if it has shown nonsusceptibility to at least one agent in three or more antimicrobial categories. Data was analysed using SPSS 16.0

\section{Results}

During the study period, a total of 46,005 samples were received for culture, from which 583 (1.35\%) Acinetobacter spp were isolated. The isolation rate of Acinetobacter spp increased from $0.7 \%$ in 2012 to $1.35 \%$ in 2013 and thereafter remaining steady till 2016. Maximum number of Acinetobacter isolates was from Sputum $(235 / 583,40 \%)$. The prevalence of MDR strains among Acinetobacter increased from 59\% in 2012 to $76 \%$ in 2016.

Antibiogram analysis of the Acinetobacter isolates showed a progressive decrease in the susceptibility to third generation Cephalosporins \& Piperacillin. Cotrimoxazole and Quinolones have showed a progressive decrease in susceptibility from 2012 to
2015, but an increased susceptibility was observed in 2016. Carbapenems, Tobramycin \& Cefoperazone-sulbactam remained the most susceptible agents. But there was a decrease in the percentage susceptibility of Imipenem, Tobramycin \& Cefoperazone-sulbactam,compared to the data from previous year.

\section{Discussion}

There has been a worldwide increase in the prevalence of Acinetobacter infections over the last two decades1. The prevalence of Acinetobacter infections in India ranges between 1.4 to $5 \%^{3,4,5} \&$ our study also shows a similar isolation rate $(1.35 \%)$. In Asia and the Middle East, resistance rates are about $40 \%$ for Ceftazidime, 35\% for Amikacin and $45 \%$ for Ciprofloxacin6. But our analysis reveals higher resistance rates for these antibiotics $(80 \%, 50 \%$ $\& 70 \%$ respectively).

Literature review shows varied resistance patterns across our country ${ }^{3-5,7}$. Carbapenem resistance rates in Acinetobacter baumanniihave generally exceeded $40 \%$ throughout all of India ${ }^{8,9,10}$. According to our study Carbapenems, Tobramycin \& Cefoperazonesulbactam are the most sensitive agents.But our study also shows a rising trend of Carbapenem resistance as Meropenem resistance rate has increased from $32 \%$ to $50 \%$ over last 5 years.

Certain studies have recommended combination therapy for Acinetobacter infections6,7.However controlled studies are needed to evaluate the efficacy of combination therapy in empirical treatment before making any formal recommendations ${ }^{1}$.

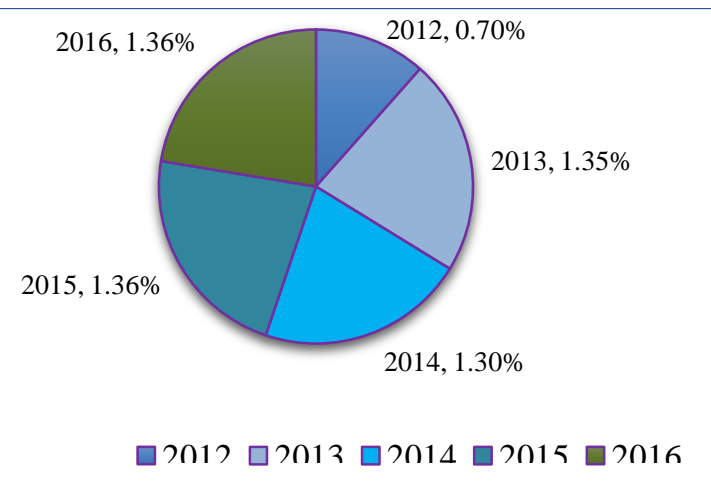

Fig. 1 Isolation rate of Acinetobacter spp from 2012-2016 


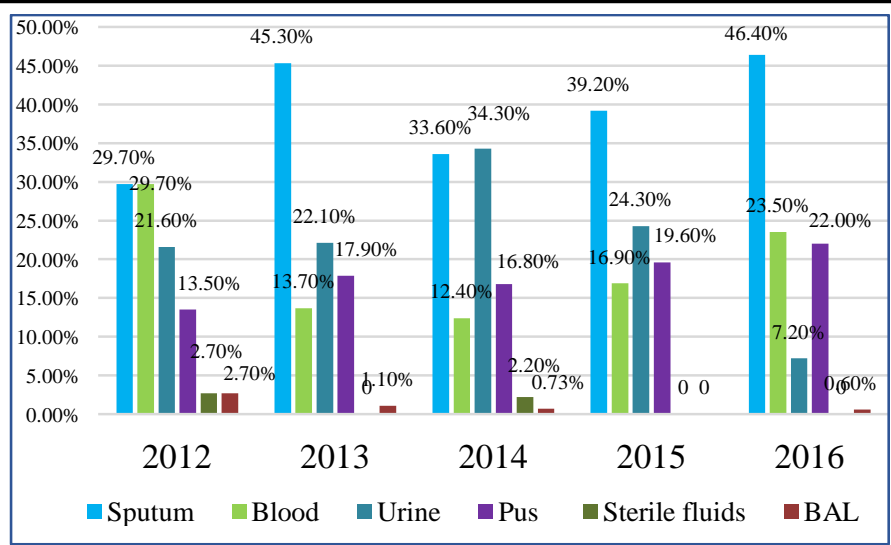

Fig. 2 Specimen Wise Isolation Rate Of Acinetobacter Spp from 2012 To 2016

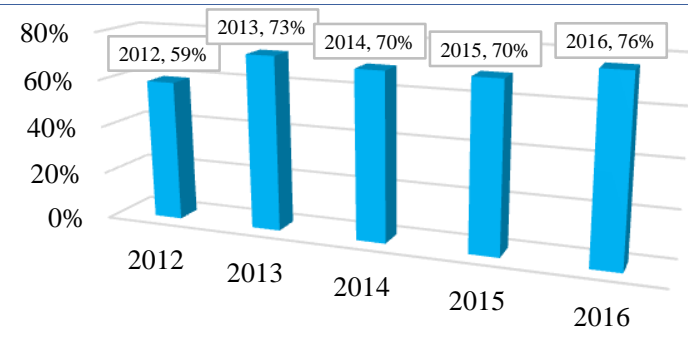

Fig. 3: Prevalence of MDR Acinetobacter from 2012 to 2016

Table 1: Percentage susceptibility of Acinetobacter spp from 2012 to 2016

\begin{tabular}{|l|c|c|c|c|c|}
\hline Antibiotics tested & 2012 & 2013 & 2014 & 2015 & 2016 \\
\hline Ceftazidime & $41 \%$ & $26 \%$ & $16 \%$ & $7 \%$ & $13 \%$ \\
\hline Gentamicin & $46 \%$ & $38 \%$ & $43 \%$ & $39 \%$ & $48 \%$ \\
\hline Piperacillin & $32 \%$ & $26 \%$ & $16 \%$ & $12 \%$ & $10 \%$ \\
\hline Pip -Tazobactam & $37 \%$ & $32 \%$ & $41 \%$ & $50 \%$ & $45 \%$ \\
\hline Cefotaxime & $16 \%$ & $13 \%$ & $4 \%$ & $5 \%$ & $3 \%$ \\
\hline Cefepime & $48 \%$ & $27 \%$ & $39 \%$ & $45 \%$ & $43 \%$ \\
\hline Amikacin & $46 \%$ & $40 \%$ & $48 \%$ & $54 \%$ & $48 \%$ \\
\hline Meropenem & $32 \%$ & $42 \%$ & $39 \%$ & $52 \%$ & $50 \%$ \\
\hline Imipenem & $*$ & $*$ & $64 \%$ & $46 \%$ & $60 \%$ \\
\hline $\begin{array}{l}\text { Cefoperazone } \\
\text { sulbactam }\end{array}$ & $*$ & $58 \%$ & $68 \%$ & $58 \%$ & $55 \%$ \\
\hline Tobramycin & $*$ & $*$ & $44 \%$ & $61 \%$ & $61 \%$ \\
\hline Cotrimoxazole & $47 \%$ & $42.6 \%$ & $39 \%$ & $28.5 \%$ & $45 \%$ \\
\hline Ciprofloxacin & $47 \%$ & $25.3 \%$ & $28 \%$ & $29.3 \%$ & $33 \%$ \\
\hline
\end{tabular}

\section{Conclusions}

MDR Acinetobacter infections in our institution have increased considerably over the past five years. The trends in the antibiotic susceptibility patterns needs to be constantly monitored as it will help to determine the emergence of drug resistance, to formulate antibiotic policies for empirical therapy and to curb the irrational usage of antibiotics. The resistance mechanism needs to be studied more as it may help in developing newer therapeutic strategies.

\section{References}

1. Mandell GL, Bennett JE, Dolin R, editors. Mandell, Douglas, and Bennett's principles and practice of infectious diseases. 7th ed. Philadelphia, PA: Churchill Livingstone/Elsevier; 2010. 2 p.

2. Kasper DL, editor. Harrison's principles of internal medicine. 19th edition / editors, Dennis L. Kasper, MD, William Ellery Channing, New York: McGraw Hill Education; 2015. 1 p

3. Agarwal J, Srivastava S, Singh M, Sinha N. Analysis of carbapenem-resistant Acinetobacter from a tertiary care setting in North India. Indian J Med Microbiol. 2013; 31(1):60.

4. Vaja DK, Kavathia DGU, Goswami DYS, Chouhan DS. A Prevalence Study of Acinetobacter Species and Their Sensitivity Pattern in a Tertiary Care Hospital Rajkot City of Gujarat (India): A Hospital Based Study. IOSR J Dent Med Sci. 2016 Jul; 15(07):54-8.

5. Sharma P, BASHIR YU, Kaur S, Kaur P, AGGARWA A. Emerging antimicrobial resistance and clinical relevance of Acinetobacter isolates in a tertiary care hospital of rural area of Punjab, India. J Microbiol Antimicrob Agents [Internet]. 2015 [cited 2017 Feb 21];1(1). Available from: http://ambs-journal.co.uk/ojs-2.4.71/index.php/JMAA/article/view/10

6. Peleg AY, Seifert H, Paterson DL. Acinetobacter baumannii: Emergence of a Successful Pathogen. Clin Microbiol Rev. 2008 Jul 1;21(3):538-82.

7. Prashanth K, Badrinath S. In vitro susceptibility pattern of Acinetobacter species to commonly used cephalosporins, 
quinolones, and aminoglycosides. Indian $\mathbf{J}$ Med Microbiol. 2004; 22(2):97.

8. Hsu L-Y, Apisarnthanarak A, Khan E, Suwantarat N, Ghafur A, Tambyah PA.

Carbapenem resistant Acinetobacter baumannii and Enterobacteriaceae in South and Southeast Asia. Clin Microbiol Rev 2017;30:1-22

9. MahajanG, Sheemar S, Chopra S, Kaur J, Chawdhary D, Makhija SK. Carbapenem resistance and phenotypic detection of carbapenemases in clinical isolates ofAcinetobacter baumannii. Indian $\mathrm{J}$ Med Sci 2011;65:18-25

10. Rynga D, Shariff M, Deb M. Phenotypic and molecular charecterization of clinical isolates of Acinetobacter baumannii isolated from Delhi, India. Ann Clin Microbiol Antimicrob 2015;14:40 